\title{
VALIDITY PROOF OF LAZARD'S METHOD FOR CAD CONSTRUCTION
}

\author{
SCOTT MCCALLUM, ADAM PARUSIŃSKI, AND LAURENTIU PAUNESCU
}

\begin{abstract}
In 1994 Lazard proposed an improved method for cylindrical algebraic decomposition $(\mathrm{CAD})$. The method comprised a simplified projection operation together with a generalized cell lifting (that is, stack construction) technique. For the proof of the method's validity Lazard introduced a new notion of valuation of a multivariate polynomial at a point. However a gap in one of the key supporting results for his proof was subsequently noticed. In the present paper we provide a complete validity proof of Lazard's method. Our proof is based on the classical parametrized version of Puiseux's theorem and basic properties of Lazard's valuation. This result is significant because Lazard's method can be applied to any finite family of polynomials, without any assumption on the system of coordinates. It therefore has wider applicability and may be more efficient than other projection and lifting schemes for CAD.
\end{abstract}

\section{INTRODUCTION}

Cylindrical algebraic decomposition (CAD) of Euclidean $n$-space $\mathbb{R}^{n}$, relative to a given set of $n$-variate integral polynomials $A$, is an important tool in computational algebra and geometry. It was introduced by Collins [15] as the key component of a method for quantifier elimination (QE) for real closed fields. Applications of CAD and other QE techniques include robot motion planning [40], stability analysis of differential equations [23, simulation and optimization [42], epidemic modelling [11], and programming with complex functions [19]. A key operation for CAD construction is projection: the projection of a set of $n$-variate integral polynomials is a set of $(n-1)$-variate integral polynomials. In view of its central role, much effort has been devoted to improving this operation [31, 32, 22, 8]. Cell lifting, or stack construction, is also an important component of CAD.

In 1994 Lazard [26] proposed an improved method for CAD computation. The method comprised a simplified projection operation together with a generalized cell lifting process. However a gap in one of the key supporting results of [26] was subsequently noticed [16, 8]. This was disappointing because Lazard's proposed approach has some advantages over other methods. In particular, it is relatively simple and it requires no assumption on the system of coordinates.

Inherent in [26] is a certain notion of valuation of a multivariate polynomial and, more generally, multivariate Laurent-Puiseux (that is fractional meromorphic) series, at a point. The related notion of the valuation-invariance of such series in a subset of $\mathbb{R}^{n}$ is also implicit in [26]. Lazard's proposed approach is in contrast with the classical approach based on the

2010 Mathematics Subject Classification. 14P10 68W30 .

Key words and phrases. Cylindrical algebraic decomposition, Lazard valuation, Puiseux with parameter theorem. 
concept of the order (of vanishing) of a multivariate polynomial or analytic function at a point, and the related concept of order-invariance, see McCallum [31, 32].

A partial validity proof of Lazard's projection only was recently published by McCallum and Hong, [36]. It was shown there that Lazard's projection is valid for CAD construction for so-called well-oriented polynomial sets. The key underlying results related to orderinvariance rather than valuation-invariance, and the validity proof was built upon established results concerning improved projection. While this was an important step forward it was only a partial validation of Lazard's approach since the method was not proved to work for non well-oriented polynomials and it did not involve valuation-invariance.

The present paper provides a complete validity proof of Lazard's method using his notion of valuation. There is no restriction of the method to well-oriented sets. This result is significant because Lazard's method has wider applicability and may be more efficient than other projection and lifting schemes for CAD. Moreover, we are hopeful that the use of Lazard's projection in CAD construction may permit greater exploitation of equational constraints, when present, in further reducing projection sets in CAD based QE [33, 34].

This paper is organised as follows. We first recall Lazard's method and main claim (Section 2). We then study the concept of Lazard's valuation (Section 3). In this paper we only consider Lazard's valuation for a multivariate polynomial. Section 4 contains the statement of a key mathematical result (the Puiseux with parameter theorem) underlying our validation of Lazard's method. In Section 5 we present our proof of Lazard's main claim using Lazard's notion of valuation. The main idea of the proof is to use monomial test curves that allow us to change the valuation invariance along an analytic submanifold to the order invariance. In the appendix at the end of the paper we present, for the reader convenience, a proof of the Puiseux with parameter theorem.

\section{LAZARD'S PROPOSED METHOD AND CLAIMS}

Background material on CAD, and in particular its projection operation, can be found in $\left[2,15,16,17,22,31,32\right.$. We present a precise definition of the projection operator $P_{L}$ for CAD introduced by Lazard [26]. Put $R_{0}=\mathbb{Z}$ and, for $n \geq 1$, put $R_{n}=R_{n-1}\left[x_{n}\right]=$ $\mathbb{Z}\left[x_{1}, \ldots, x_{n}\right]$. Elements of the ring $R_{n}$ will usually be considered to be polynomials in $x_{n}$ over $R_{n-1}$. We shall call a subset $A$ of $R_{n}$ whose elements are irreducible polynomials of positive degree and pairwise relatively prime an irreducible basis. (This concept is analogous to that of squarefree basis which is used in the CAD literature, for example [31].)

Definition 2.1 (Lazard projection). Let $A$ be a finite irreducible basis in $R_{n}$, with $n \geq 2$. The Lazard projection $P_{L}(A)$ of $A$ is the subset of $R_{n-1}$ comprising the following polynomials:

(1) all leading coefficients of the elements of $A$,

(2) all trailing coefficients (i.e. coefficients independent of $x_{n}$ ) of the elements of $A$,

(3) all discriminants of the elements of $A$, and

(4) all resultants of pairs of distinct elements of $A$.

Remark 2.2. Lazard's projection could alternatively be defined for a (slightly modified) squarefree basis $A$ in $R_{n}$, as in [26]. We use an irreducible basis in our definition because experience has shown that this likely leads to a more efficient CAD algorithm in practice and on average. 
Remark 2.3. Let $A$ be an irreducible basis. Lazard's projection $P_{L}(A)$ is contained in and is usually strictly smaller than the McCallum projection $P_{M}(A)$ 31, 32. Indeed $P_{M}(A)$ includes the "middle coefficients" (i.e. those coefficients other than the leading and trailing ones) of the elements of $A$, which $P_{L}(A)$ omits. In other respects these two projection operators are the same. However $P_{L}(A)$ contains and is usually strictly larger than the Brown-McCallum projection $P_{B M}(A)$ [8]. Indeed $P_{B M}(A)$ omits the trailing coefficients of the elements of $A$, which $P_{L}(A)$ includes, but in other respects is the same as $P_{L}(A)$. This remark notwithstanding, the Lazard projection is still of interest because of certain limitations of the Brown-McCallum projection. The two chief drawbacks of the projection $P_{B M}(A)$ are as follows. First, the method of [8] could fail in case $A$ is not well-oriented [32, 8]. Second, the method requires that any 0-dimensional nullifying cells [32, 8$]$ in each dimension be identified and added during CAD construction. These drawbacks are elaborated in [8].

Lazard [26] outlined a claimed CAD algorithm for $A \subset R_{n}$ and $\mathbb{R}^{n}$ which uses the projection set $P_{L}(A)$. The specification of his algorithm requires the following concept of his valuation:

Definition 2.4 (Lazard valuation). Let $K$ be a field. Let $n \geq 1, f \in K\left[x_{1}, \ldots, x_{n}\right]$ nonzero, and $\alpha=\left(\alpha_{1}, \ldots, \alpha_{n}\right) \in K^{n}$. The Lazard valuation (valuation, for short) $v_{\alpha}(f)$ of $f$ at $\alpha$ is the element $\mathbf{v}=\left(v_{1}, \ldots, v_{n}\right)$ of $\mathbb{N}^{n}$ least (with respect to $\leq_{\text {lex }}$ ) such that $f$ expanded about $\alpha$ has a term

$$
c\left(x_{1}-\alpha_{1}\right)^{v_{1}} \cdots\left(x_{n}-\alpha_{n}\right)^{v_{n}}
$$

with $c \neq 0$. (Note that $\leq_{\text {lex }}$ denotes the lexicographic order on $\mathbb{N}^{n}$ - see next section.)

Example 2.5. Let $n=1$. Then $v_{\alpha}(f)$ is the familiar order $\operatorname{ord}_{\alpha}(f)$ of $f \in K\left[x_{1}\right]$ at $\alpha \in K$. Thus, for instance, if $f\left(x_{1}\right)=x_{1}^{2}-x_{1}^{3}$ then $v_{0}(f)=2$ and $v_{1}(f)=1$. As another example, let $n=2$ and $f\left(x_{1}, x_{2}\right)=x_{1} x_{2}^{2}+x_{1}^{2} x_{2}=x_{1} x_{2}\left(x_{2}+x_{1}\right)$. Then $v_{(0,0)}(f)=(1,2), v_{(1,0)}(f)=(0,1)$, and $v_{(0,1)}(f)=(1,0)$.

The above defines $v_{\alpha}(f)$ for $f \in K\left[x_{1}, \ldots, x_{n}\right]$ nonzero and $\alpha \in K^{n}$. Lazard [26] actually defined $v_{\alpha}(f)$ for nonzero elements $f$ of the much larger domain of all Laurent-Puiseux (that is, fractional meromorphic) series in $x_{1}-\alpha_{1}, \ldots, x_{n}-\alpha_{n}$ over $K$. In this sense the above is a more limited definition of valuation. With $K, n$ and $f$ as in the above definition, and $S \subset K^{n}$, we say $f$ is valuation-invariant in $S$ if the valuation of $f$ is the same at every point of $S$. Some basic properties of this Lazard valuation, and the associated notion of valuation-invariance, are presented in Section 3 below. Lazard's proposed CAD algorithm also uses a technique for "evaluating" a polynomial $f \in R_{n}$ at a sample point in $\mathbb{R}^{n-1}$. This technique is described in slightly more general terms as follows:

Definition 2.6 (Lazard evaluation). Let $K$ be a field which supports explicit arithmetic computation. Let $n \geq 2$, take a nonzero element $f$ in $K\left[x_{1}, \ldots, x_{n}\right]$, and let $\alpha=\left(\alpha_{1}, \ldots, \alpha_{n-1}\right) \in$ $K^{n-1}$. The Lazard evaluation $f_{\alpha}\left(x_{n}\right) \in K\left[x_{n}\right]$ of $f$ at $\alpha$ is defined to be the result of the following process (which determines also nonnegative integers $v_{i}$, with $1 \leq i \leq n-1$ ):

$$
\begin{aligned}
& f_{\alpha} \leftarrow f \\
& \text { For } i \leftarrow 1 \text { to } n-1 \text { do } \\
& \quad v_{i} \leftarrow \text { the greatest integer } v \text { such that }\left(x_{i}-\alpha_{i}\right)^{v} \mid f_{\alpha} \\
& \quad f_{\alpha} \leftarrow f_{\alpha} /\left(x_{i}-\alpha_{i}\right)^{v_{i}}
\end{aligned}
$$




$$
f_{\alpha} \leftarrow f_{\alpha}\left(\alpha_{i}, x_{i+1}, \ldots, x_{n}\right)
$$

Example 2.7. We illustrate the above evaluation method using two simple examples. For both examples we take $K=\mathbb{Q}, n=3$ and $\alpha=(0,0)$. We denote $\left(x_{1}, x_{2}, x_{3}\right)$ by $(x, y, z)$. First let $f(x, y, z)=z^{2}+y^{2}+x^{2}-1$. After the first pass through the method $(i=1)$ we have $v_{1}=0$ and $f_{\alpha}(y, z)=z^{2}+y^{2}-1$. After the second pass $(i=2)$ we have $v_{2}=0$ and $f_{\alpha}(z)=z^{2}-1$. In this case $f_{\alpha}(z)=f(0,0, z)$. For our second example let $f(x, y, z)=y z-x$. After the first pass $(i=1)$ we have $v_{1}=0$ and $f_{\alpha}(y, z)=y z$. After the second pass $(i=2)$ we have $v_{2}=1$ and $f_{\alpha}(z)=y z / y=z$. In this case $f_{\alpha}(z) \neq f(0,0, z)$, because the latter polynomial is zero.

Remark 2.8. Notice that the assertion " $f_{\alpha} \neq 0$ " is an invariant of the above process. With $K, n, f, \alpha$ and the $v_{i}$ as in the above definition of Lazard evaluation, notice that $f\left(\alpha, x_{n}\right)=0$ (identically) if and only if $v_{i}>0$, for some $i$ in the range $1 \leq i \leq n-1$. With $\alpha_{n} \in K$ arbitrary, notice also that the integers $v_{i}$, with $1 \leq i \leq n-1$, are the first $n-1$ coordinates of $v_{\left(\alpha, \alpha_{n}\right)}(f)$. It will on occasion be handy to refer to the $(n-1)$-tuple $\left(v_{1}, \ldots, v_{n-1}\right)$ as the Lazard valuation of $f$ on $\alpha$.

Remark 2.9. Let $f \in K\left[x_{1}, \ldots, x_{n}\right]$ be nonzero, $\alpha=\left(\alpha_{1}, \ldots, \alpha_{n-1}\right) \in K^{n-1}$, and let $\mathbf{v}=$ $\left(v_{1}, \ldots, v_{n-1}\right)$ be the Lazard valuation of $f$ on $\alpha$. If we expand $f$ at $\alpha$

$$
f\left(x_{1}, \ldots, x_{n}\right)=\sum_{u} f^{\mathbf{u}}\left(x_{n}\right) \prod_{i=1}^{n-1}\left(x_{i}-\alpha_{i}\right)^{u_{i}}
$$

where $\mathbf{u}=\left(u_{1}, \ldots, u_{n-1}\right) \in \mathbb{N}^{n-1}$, with coefficients $f^{\mathbf{u}}\left(x_{n}\right) \in K\left[x_{n}\right]$, then $f_{\alpha}=f^{\mathbf{v}}$. This follows from the fact that $\mathbf{v}$ is the minimum of $\left\{\mathbf{u}: f^{\mathbf{u}} \neq 0\right\}$ for the lexicographic order.

One more definition is needed before we can state Lazard's main claim and his algorithm based on it. This definition is not explicit in [26] - it was introduced in [36] to help clarify and highlight Lazard's main claim:

Definition 2.10. [Lazard delineability] With $K=\mathbb{R}$ and $x$ denoting $\left(x_{1}, \ldots, x_{n-1}\right)$, let $f$ be a nonzero element of $\mathbb{R}\left[x, x_{n}\right]$ and $S$ a subset of $\mathbb{R}^{n-1}$. We say that $f$ is Lazard delineable on $S$ if

(1) the Lazard valuation of $f$ on $\alpha$ is the same for each point $\alpha \in S$;

(2) there exist finitely many continuous functions $\theta_{1}<\cdots<\theta_{k}$ from $S$ to $\mathbb{R}$, with $k \geq 0$, such that, for all $\alpha \in S$, the set of real roots of $f_{\alpha}\left(x_{n}\right)$ is $\left\{\theta_{1}(\alpha), \ldots, \theta_{k}(\alpha)\right\}$ (where in case $k=0$, this means that, for all $\alpha \in S$, the set of real roots of $f_{\alpha}\left(x_{n}\right)$ is empty); and in case $k>0$

(3) there exist positive integers $m_{1}, \ldots, m_{k}$ such that, for all $\alpha \in S$ and all $i, m_{i}$ is the multiplicity of $\theta_{i}(\alpha)$ as a root of $f_{\alpha}\left(x_{n}\right)$.

When $f$ is Lazard delineable on $S$ we refer to the graphs of the $\theta_{i}$ as the Lazard sections of $f$ over $S$, and to the $m_{i}$ as the associated multiplicities of these sections. The regions between successive Lazard sections, together with the region below the lowest Lazard section and that above the highest Lazard section, are called Lazard sectors.

Proposition 2.11. If $f$ is Lazard delineable on $S$ then $f$ is valuation-invariant in every Lazard section and sector of $f$ over $S$. 
Proof. Let $\left(v_{1}, \ldots, v_{n-1}\right)$ be the common value of the Lazard valuation of $f$ on $\alpha \in S$. Then $\left(v_{1}, \ldots, v_{n-1}, 0\right)$ is the Lazard valuation of $f$ at a point $(\alpha, z)$ in a Lazard sector of $f$ over $S$. If $(\alpha, z)$ is in a Lazard section of $f$ over $S$ with associated multiplicity $m$ then the Lazard valuation of $f$ at this point equals $\left(v_{1}, \ldots, v_{n-1}, m\right)$.

We express Lazard's main claim, essentially the content of his Proposition 5 and subsequent remarks, as follows (as in [36]):

Let $A$ be a finite irreducible basis in $R_{n}$, where $n \geq 2$. Let $S$ be a connected subset of $\mathbb{R}^{n-1}$. Suppose that each element of $P_{L}(A)$ is valuation-invariant in $S$. Then each element of $A$ is Lazard delineable on $S$, and the Lazard sections over $S$ of the elements of $A$ are pairwise disjoint.

Our wording of this claim is different from Lazard's - we have tried to highlight and clarify the essence of his assertions. This claim concerns valuation-invariant lifting in relation to $P_{L}(A)$ : it asserts that the condition, "each element of $P_{L}(A)$ is valuation-invariant in $S$ ", is sufficient for an $A$-valuation-invariant stack in $\mathbb{R}^{n}$ to exist over $S$. We prove Lazard's main claim in Section 5. We can now describe Lazard's proposed CAD algorithm (as in [36]):

Algorithm 2.12 (Valuation-invariant CAD using Lazard projection).

$$
(\mathcal{I}, \mathcal{S}) \leftarrow \operatorname{VCADL}(A)
$$

Input: $A$ is a list of integral polynomials in $x_{1}, \ldots, x_{n}$.

Output: $\mathcal{I}$ and $\mathcal{S}$ are lists of indices and sample points, respectively, of the cells comprising an $A$-valuation-invariant $\mathrm{CAD}$ of $\mathbb{R}^{n}$.

(1) If $n>1$ then go to (2).

Isolate the real roots of the irreducible factors of the nonzero elements of $A$. (Algorithms for univariate integral polynomial factorization are given in [25].) Construct cell indices $\mathcal{I}$ and sample points $\mathcal{S}$ from the real roots. Exit.

(2) $B \leftarrow$ the finest squarefree basis for $\operatorname{prim}(A)$. That is, $B$ is assigned the set of ample irreducible factors of elements of the set $\operatorname{prim}(A)$ of primitive parts of elements of $A$ of positive degree. (Recall that an ample set in a commutative ring with 1 is a set which contains exactly one element in each equivalence class of associates [14, 15. Algorithms for multivariate integral polynomial factorization are given in [25].) $P \leftarrow \operatorname{cont}(A) \cup P_{L}(B)$. $(\operatorname{cont}(A)$ denotes the set of contents of elements of $A$.) $\left(\mathcal{I}^{\prime}, \mathcal{S}^{\prime}\right) \leftarrow \operatorname{VCADL}(P)$.

$\mathcal{I} \leftarrow$ the empty list. $\mathcal{S} \leftarrow$ the empty list.

For each $\alpha=\left(\alpha_{1}, \ldots, \alpha_{n-1}\right)$ in $\mathcal{S}^{\prime}$ do

Let $i$ be the index of the cell containing $\alpha$.

$f^{*} \leftarrow \prod_{f \in B} f_{\alpha}$. (Each $f_{\alpha}$ is constructed using exact arithmetic in $\mathbb{Q}(\alpha)$.)

Isolate the real roots of $f^{*}$.

Construct cell indices and sample points for Lazard sections and sectors of elements of $B$ from $i, \alpha$ and the real roots of $f^{*}$.

Add the cell indices to $\mathcal{I}$ and the sample points to $\mathcal{S}$.

Exit. 
As mentioned in Remark 2.2, practical experience with CAD suggests that it is worthwhile overall to first compute the finest squarefree basis for $\operatorname{prim}(A)$ in step $(2)$. In particular, the cost in practice of such computation usually remains a relatively small part of the total time for CAD. But the benefit of such computation as the projection and lifting phases of CAD proceed likely outweighs any additional cost of performing the factorization. This is due in part to the expected presence of nontrivial factors of repeated discriminants and resultants [27, 12].

The correctness of the above algorithm - namely, the claim that, given $A \subset R_{n}$, it produces a CAD of $\mathbb{R}^{n}$ such that each cell of the CAD is valuation-invariant with respect to each element of $A$ - follows from Lazard's main claim by induction on $n$.

\section{BASIC PROPERTIES OF LAZARD's VALUATION}

In this section we study Lazard's valuation [26] in the relatively special setting, namely that of multivariate polynomials over a ring, in which it was defined in the previous section. We shall clarify the notion and basic properties of this special valuation, and identify some relationships between valuation-invariance and order-invariance. The content of this section is based on very similar material found in [35].

We recall the standard algebraic definition of the term valuation [18, 3, 48, A mapping $v: R-\{0\} \rightarrow \Gamma, R$ a ring, into a totally ordered abelian monoid (written additively) $\Gamma$ is said to be a valuation of $R$ if the following two conditions are satisfied:

(1) $v(f g)=v(f)+v(g)$ for all $f$ and $g$;

(2) $v(f+g) \geq \min \{v(f), v(g)\}$, for all $f$ and $g$ (with $f+g \neq 0$ ).

Perhaps the simplest and most familiar example of a valuation in algebraic geometry is the order of an $n$-variate polynomial over a field $K$ at a point $\alpha \in K^{n}$. That is, the mapping $\operatorname{ord}_{\alpha}: K\left[x_{1}, \ldots, x_{n}\right]-\{0\} \rightarrow \mathbb{N}$ defined by

$$
\operatorname{ord}_{\alpha}(f)=\text { the order of } f \text { at } \alpha
$$

is a valuation of the ring $K\left[x_{1}, \ldots, x_{n}\right]$. The order of $f$ at $\alpha$ is also called "the multiplicity of $f$ at $\alpha "$.

Let $n \geq 1$. Recall that the lexicographic order $\leq_{l e x}$ on $\mathbb{N}^{n}$ is defined by $v=\left(v_{1}, \ldots, v_{n}\right) \leq_{l e x}$ $\left(w_{1}, \ldots, w_{n}\right)=w$ if and only if either $v=w$ or there is some $i, 1 \leq i \leq n$, with $v_{j}=w_{j}$, for all $j$ in the range $1 \leq j<i$, and $v_{i}<w_{i}$. Then $\leq_{l e x}$ is an admissible order on $\mathbb{N}^{n}$ in the sense of [5]. Indeed $\mathbb{N}^{n}$, together with componentwise addition and $\leq_{l e x}$, forms a totally ordered abelian monoid. The lexicographic order $\leq_{l e x}$ can be defined similarly on $\mathbb{Z}^{n}$, forming a totally ordered abelian group.

Recall the definition of $v_{\alpha}(f)$ for $f \in K\left[x_{1}, \ldots, x_{n}\right]$ nonzero and $\alpha \in K^{n}$ from Section 2: $v_{\alpha}(f)$ is the element $\left(v_{1}, \ldots, v_{n}\right)$ of $\mathbb{N}^{n}$ least (with respect to $\leq_{\text {lex }}$ ) such that $f$ expanded about $a$ has a term $c\left(x_{1}-\alpha_{1}\right)^{v_{1}} \cdots\left(x_{n}-\alpha_{n}\right)^{v_{n}}$ with $c \neq 0$. Notice that $v_{\alpha}(f)=(0, \ldots, 0)$ if and only if $f(\alpha) \neq 0$. Where there is no ambiguity we shall usually omit the qualifier "Lazard" from "Lazard valuation". We state some basic properties of the valuation $v_{\alpha}(f)$, analogues of properties of the familiar order $\operatorname{ord}_{\alpha}(f)$. The first property is the fulfilment of the axioms. 
Proposition 3.1. Let $f$ and $g$ be nonzero elements of $K\left[x_{1}, \ldots, x_{n}\right]$ and let $\alpha \in K^{n}$. Then $v_{\alpha}(f g)=v_{\alpha}(f)+v_{\alpha}(g)$ and $v_{\alpha}(f+g) \geq_{\text {lex }} \min \left\{v_{\alpha}(f), v_{\alpha}(g)\right\}$ (if $\left.f+g \neq 0\right)$.

Proof. These claims follow since $\mathbb{N}^{n}$, together with componentwise addition and $\leq_{l e x}$, forms a totally ordered abelian monoid.

Proposition 3.2. (Upper semicontinuity of valuation) Let $f$ be a nonzero element of $K\left[x_{1}, \ldots, x_{n}\right]$ and let $v=\left(v_{1}, \ldots, v_{n}\right) \in \mathbb{N}^{n}$. Then the set $\left\{\gamma \in K^{n} ; v_{\gamma}(f) \geq_{\text {lex }} v\right\}$ is an algebraic subset of $K^{n}$. In particular, the Lazard valuation is upper semi-continuous (in Zariski topology for any field $K$ and in the classical topology for $K=\mathbb{R}$ or $\mathbb{C}$ ).

Proof. Denote $w=\left(w_{1}, \ldots, w_{n}\right), \alpha=\left(\alpha_{1}, \ldots, \alpha_{n}\right)$. The coefficient $c_{w, \alpha}$ in the expansion of $f$ at $\alpha$

$$
f=\sum_{w} c_{w, \alpha}\left(x_{1}-\alpha_{1}\right)^{w_{1}} \cdots\left(x_{n}-\alpha_{n}\right)^{w_{n}},
$$

for $w$ fixed, is a polynomial in $\alpha$. (If $K$ is of characteristic zero then this coefficient equals $\left.\frac{1}{w_{1} ! \cdots w_{n} !} \frac{\partial^{w_{1}+\cdots+w_{n}} f}{\partial x_{1}^{w_{1}} \cdots \partial x_{n}^{w_{n}}}.\right)$ The set $\left\{\alpha \in K^{n} ; v_{\alpha}(f) \geq_{\text {lex }} v\right\}$ is the intersection of the zero set of polynomials $c_{w, \alpha}$ for $w=\left(w_{1}, \ldots, w_{n}\right)<_{l e x} v$. Therefore it is algebraic. The algebraic sets are closed in Zariski topology by definition and clearly also, if $K=\mathbb{R}$ or $\mathbb{C}$, in the classical topology.

Remark 3.3. Let $f: U \rightarrow K, K=\mathbb{R}$ or $\mathbb{C}$, be analytic, where $U$ is an open connected subset of $K^{n}$, and suppose that $f$ does not vanish identically. Then the Lazard valuation $v_{\alpha}(f)$ for $\alpha \in U$ can be defined exactly as in Definition 2.4 and it satisfies the upper semicontinutiy property for the classical topology. But the Lazard valuation extended to rational or meromorphic functions does not satisfy the upper semicontinutiy, see [35] for a discussion.

We shall say that $f$ is valuation-invariant in a subset $S \subset K^{n}$ if $v_{\alpha}(f)$ is constant as $\alpha$ varies in $S$.

For the remaining properties we state we shall assume that $K=\mathbb{R}$ or $\mathbb{C}$. (We will use Proposition 3.4 later but not the remaining properties.)

Proposition 3.4. Let $f$ and $g$ be analytic in $U \subset K^{n}$, with $U$ open and connected, and suppose neither $f$ nor $g$ vanishes identically. (In particular, $f$ and $g$ could be nonzero elements of $K\left[x_{1}, \ldots, x_{n}\right]$, with $U=K^{n}$.) Let $S \subset U$ be connected. Then $f g$ is valuationinvariant in $S$ if and only if both $f$ and $g$ are valuation-invariant in $S$.

Proof. This follows easily from Proposition 3.2 and Remark 3.3 . See for instance the proof of Lemma A.3 of [31].

The next lemma is in a sense another analogue of the familiar order, and is particular to the case $n=2$.

Lemma 3.5. Let $f(x, y) \in K[x, y]$ be primitive of positive degree in $y$ and squarefree. Then for all but a finite number of points $(\alpha, \beta) \in K^{2}$ on the curve $f(x, y)=0$ we have $v_{(\alpha, \beta)}(f)=$ $(0,1)$. 
Proof. Denote by $R(x)$ the resultant $\operatorname{res}_{y}\left(f, f_{y}\right)$ of $f$ and $f_{y}$ with respect to $y$. Then $R(x) \neq 0$ since $f$ is assumed squarefree. Let $(\alpha, \beta) \in K^{2}$, suppose $f(\alpha, \beta)=0$ and assume that $v_{(\alpha, \beta)}(f) \neq(0,1)$. Then $f_{y}(\alpha, \beta)=0$. Hence $R(\alpha)=0$. So $\alpha$ belongs to the set of roots of $R(x)$, a finite set. Now $f(\alpha, \beta)=0$ and $f(\alpha, y) \neq 0$, since $f$ is assumed primitive. So $\beta$ belongs to the set of roots of $f(\alpha, y)$, a finite set.

Let us further consider the relationship between the concepts of order-invariance and valuation-invariance for a subset $S$ of $K^{n}$. The concepts are the same in case $n=1$ because order and valuation are the same for this case. For $n=2$ order-invariance in $S$ does not imply valuation-invariance in $S$. (For consider $S=\left\{(x, y) \mid x^{2}+y^{2}-1=0\right\}$ in $K^{2}$. The order of $f(x, y)=x^{2}+y^{2}-1$ at every point of $S$ is 1 . The valuation of $f$ at every point $(\alpha, \beta) \in S$ except $( \pm 1,0)$ is $(0,1)$. But $v_{( \pm 1,0)}(f)=(0,2)$.) However for $n=2$ we can prove the following.

Proposition 3.6. Let $f \in K[x, y]$ be nonzero and $S \subset K^{2}$ be connected. If $f$ is valuationinvariant in $S$ then $f$ is order-invariant in $S$.

Proof. Assume that $f$ is valuation-invariant in $S$. Write $f$ as a product of irreducible elements $f_{i}$ of $K[x, y]$. By Proposition 3.4 each $f_{i}$ is valuation-invariant in $S$. We shall show that each $f_{i}$ is order-invariant in $S$. Take an arbitrary factor $f_{i}$. If the valuation of $f_{i}$ in $S$ is $(0,0)$ then the order of $f_{i}$ throughout $S$ is 0 , hence $f_{i}$ is order-invariant in $S$. So we may assume that the valuation of $f_{i}$ is nonzero in $S$, that is, that $S$ is contained in the curve $f_{i}(x, y)=0$. Suppose first that $f_{i}$ has positive degree in $y$. Now the conclusion is immediate in case $S$ is a singleton, so assume that $S$ is not a singleton. Since $S$ is connected, $S$ is an infinite set. By Lemma 3.5 and valuation-invariance of $f_{i}$ in $S$, we must have $v_{(\alpha, \beta)}\left(f_{i}\right)=(0,1)$ for all $(\alpha, \beta) \in S$. Hence $f_{i}$ is order-invariant in $S$ (since ord $f_{i}=1$ in $\left.S\right)$. Suppose instead that $f_{i}=f_{i}(x)$ has degree 0 in $y$. Since $f_{i}(x)$ is irreducible it has no multiple roots. Therefore $v_{(\alpha, \beta)}\left(f_{i}\right)=(1,0)$ for all $(\alpha, \beta) \in S$. Hence $f_{i}$ is order-invariant in $S$ (since ord $f_{i}=1$ in $S$ ). The proof that $f_{i}$ is order-invariant in $S$ is finished and we conclude that $f$ is order-invariant in $S$.

However the following example indicates that Proposition 3.6 is not true for dimension greater than 2; that is, valuation-invariance does not imply order-invariance when $n>2$. Let $f(x, y, z)=z^{2}-x y$ and let $S$ be the $x$-axis in $\mathbb{R}^{3}$. Now $f$ is valuation-invariant in $S$, since the valuation of $f$ at each point of $S$ is $(0,0,2)$. But $f$ is not order-invariant in $S$, since $\operatorname{ord}_{(0,0,0)} f=2$ and $\operatorname{ord}_{(\alpha, 0,0)} f=1$ for $\alpha \neq 0$.

\section{The Puiseux With Parameter theorem}

We recall the classical Puiseux with parameter theorem in the form given in [39]. This theorem is a special case of the Abhyankar-Jung theorem, see [1], [38], and hence can be traced back to [24]. Puiseux with parameter is closely related to certain algebraic results of Zariski concerning equisingularity in codimension 1 over an arbitrary algebraically closed field of characteristic 0 (Thm 7 of [45] and Thms 4.4 and 4.5 of [46]). In the Appendix at the end of this paper we provide a short proof of this theorem for the reader's convenience.

We use the following notation: with $\varepsilon=\left(\varepsilon_{1}, \ldots, \varepsilon_{k}\right), U_{\varepsilon, r}=U_{\varepsilon} \times U_{r}$, where $U_{\varepsilon}=\{x=$ $\left.\left(x_{1}, \ldots, x_{k}\right) \in \mathbb{C}^{k}:\left|x_{i}\right|<\varepsilon_{i}, \forall i\right\}, U_{r}=\{y \in \mathbb{C}:|y|<r\}$. In this section "analytic" means "complex analytic". 
Theorem 4.1. (Puiseux with parameter)

Let

$$
f(x, y, z)=z^{d}+\sum_{i=0}^{d-1} a_{i}(x, y) z^{i}
$$

be a monic polynomial in $z$ with coefficients $a_{i}(x, y)$ analytic in $U_{\varepsilon, r}$. Suppose that the discriminant of $f$ is of the form $D_{f}(x, y)=y^{m} u(x, y)$ with analytic function $u$ non vanishing on $U_{\varepsilon, r}$. Then, there are a positive integer $N$ (we may take $N=d$ !) and analytic functions $\xi_{i}(x, t): U_{\varepsilon, r^{1 / N}} \rightarrow \mathbb{C}$ such that

$$
f\left(x, t^{N}, z\right)=\prod_{i=1}^{d}\left(z-\xi_{i}(x, t)\right)
$$

for all $(x, t) \in U_{\varepsilon, r^{1 / N}}$.

The roots $\xi_{i}(x, t)$ satisfy, moreover, the following properties. Firstly, for every $i \neq j, \xi_{i}-\xi_{j}$ equals a power of $t$ times a function nonvanishing on $U_{\varepsilon, r^{1 / N}}$. Secondly, if the coefficients $a_{i}(x, y)$ of $f$ are (complexifications of) real analytic functions then the set of functions $\xi_{i}(x, t)$ is complex conjugation invariant.

Note that "parameter" in the name "Puiseux with parameter" refers to the $k$-tuple $x$, which "parametrizes" the Puiseux roots $\xi_{i}(x, t)$. Observe also that Theorem 4.1 is applicable to any nonmonic polynomial $f(x, y, z)$ which otherwise satisfies the hypotheses of the theorem, and for which the leading coefficient $a_{d}(x, y)$ vanishes nowhere in $U_{\varepsilon, r}$. For we may simply divide $f$ by $a_{d}$ to obtain a monic polynomial with the same roots which still satisfies the hypotheses of the theorem.

By analogy with 36] we now consider the more general case in which

$$
f(x, y, z)=a_{d}(x, y) z^{d}+\sum_{i=0}^{d-1} a_{i}(x, y) z^{i},
$$

with coefficients $a_{i}(x, y)$ analytic in $U_{\varepsilon, r}$, under the assumption that each of $a_{d}(x, y)$ and $D_{f}(x, y)$ is equal to a power of $y$ times a unit, that is, an analytic function nonvanishing in $U_{\varepsilon, r}$. We may then apply Theorem 4.1 to $\tilde{f}(x, y, \tilde{z})$ defined by

$$
\tilde{f}(x, y, \tilde{z})=\tilde{z}^{d}+\sum_{i=0}^{d-1} a_{i} a_{d}^{d-1-i} \tilde{z}^{i}
$$

because $D_{\tilde{f}}=a_{d}^{d^{2}-3 d+2} D_{f}$ satisfies the assumptions of Theorem 4.1. Let $\eta_{i}(x, t)$ be the roots of $\tilde{f}\left(x, t^{N}, \tilde{z}\right)$. Then, with $y=t^{N}$,

$$
\prod_{i=1}^{d}\left(a_{d} z-\eta_{i}\right)=\tilde{f}\left(x, y, a_{d} z\right)=a_{d}^{d-1} f(x, y, z) .
$$

The above relation is key for proving the following result, which is a complex analytic version of Corollary 3.15 of [36]. 
Corollary 4.2. Let $f(x, y, z)$ be as in (3) and suppose that each of $a_{d}(x, y), a_{0}(x, y)$ and $D_{f}(x, y)$ is of the form a power of $y$ times an analytic function nonvanishing on $U_{\varepsilon, r}$. Then there are an integer $N>0$, analytic functions $u_{i}(x, t): U_{\varepsilon, r^{1 / N}} \rightarrow \mathbb{C}, 1 \leq i \leq d$ and non-negative integers $m_{0}, m_{1}, \ldots, m_{d}$, such that on $U_{\varepsilon, r^{1 / N}}$,

$$
t^{d m_{0}} f\left(x, t^{N}, z\right)=a_{d}\left(x, t^{N}\right) \prod_{i=1}^{d}\left(t^{m_{0}} z-t^{m_{i}} u_{i}(x, t)\right) .
$$

The functions $u_{i}(x, t)$ satisfy, moreover, the following properties. For all $i, u_{i}$ is nonvanishing in $U_{\varepsilon, r^{1 / N}}$. For every $i \neq j, t^{m_{i}} u_{i}-t^{m_{j}} u_{j}$ equals a power of $t$ times a function nonvanishing on $U_{\varepsilon, r^{1 / N}}$. If the coefficients of $a_{i}(x, y)$ of $f$ are (complexifications of) real analytic functions then the set of functions $u_{i}(x, t)$ is complex conjugation invariant.

Proof. By the assumption $a_{d}\left(x, t^{N}\right)=t^{m_{0}} \tilde{a}_{d}\left(x, t^{N}\right)$ and $a_{0}\left(x, t^{N}\right)=t^{m} \tilde{a}_{0}\left(x, t^{N}\right)$, with $\tilde{a}_{d}, \tilde{a}_{0}$ nowhere vanishing. Since

$$
\prod_{i} \eta_{i}\left(x, t^{N}\right)=a_{d}^{d-1}\left(x, t^{N}\right) a_{0}\left(x, t^{N}\right)
$$

the same is true for each $\eta_{i}$ (the roots of $\tilde{f}$ ), namely, $\eta_{i}(x, t)=t^{m_{i}} \tilde{\eta}_{i}(x, t)$, with $\tilde{\eta}_{i}(x, t)$ nowhere vanishing. By (5) we get $\tilde{a}_{d}^{d} \prod_{i=1}^{d}\left(t^{m_{0}} z-\tilde{a}_{d}^{-1} \eta_{i}\right)=\tilde{a}_{d}^{d} \prod_{i=1}^{d}\left(t^{m_{0}} z-t^{m_{i}} \tilde{a}_{d}^{-1} \tilde{\eta}_{i}\right)=a_{d}^{d-1} f(x, y, z)$. Hence, setting $u_{i}:=\tilde{a}_{d}^{-1}\left(x, t^{N}\right) \tilde{\eta}_{i}(x, t)$ we obtain the required formula for $t^{d m_{0}} f\left(x, t^{N}, z\right)$. If the coefficients $a_{i}$ are real then the set of roots $\eta_{i}$ is conjugation invariant and hence so is the set of functions $u_{i}(x, t)$

Remark 4.3. By analogy with Corollary 3.15 of [36], just the hypotheses on the leading coefficient $a_{d}(x, y)$ and discriminant $D_{f}(x, y)$ of a nonmonic polynomial $f(x, y, z)$ suffice to yield a conclusion slightly weaker than that presented in Corollary 4.2 above. In particular, the existence of integer $N>0$, analytic functions $u_{i}(x, t)$ and nonnegative integers $m_{i}$ yielding a factorization of $t^{d m_{0}} f\left(x, t^{N}, z\right)$ follow from just these hypotheses. Formally, the roots $t^{m_{i}-m_{0}} u_{i}(x, t)$ of $f\left(x, t^{N}, z\right)$ are meromorphic (Laurent) series: this means that finitely many negative exponents in $t$, as well as $u_{i} \equiv 0$, are allowed. Hence some roots could go to infinity as $t \rightarrow 0$, where such behaviour in general depends on the parameter $x$. (With $k=1$, consider the example $f(x, y, z)=y z-x$.) The additional hypothesis of Corollary 4.2 above is that the trailing coefficient $a_{0}(x, y)$ is of the same special form as $a_{d}(x, y)$ and $D_{f}(x, y)$. This hypothesis on $a_{0}(x, y)$ is key to proving the nonvanishing of the functions $u_{i}(x, t)$ in $U_{\varepsilon, r^{1 / N}}$, which is not guaranteed without it (as the example shows). The nonvanishing of the $u_{i}(x, t)$ implies, in turn, that the number of roots which tend to infinity as $t \rightarrow 0$ is independent of $x$. This consequence is crucial to part of the proof of our main theorem presented in the next section.

As follows from the next lemma the assumptions of Theorem 4.1 and Corollary 4.2 can be expressed equivalently as the order invariance of $a_{d}(x, y), a_{0}(x, y)$ and $D_{f}(x, y)$ in the hyperplane $y=0$.

Lemma 4.4. Recall that $x$ denotes $\left(x_{1}, \ldots, x_{k}\right)$ in this section. Let $g(x, y)$ be analytic in a neighbourhood of the origin in $K^{k+1}, K=\mathbb{R}$ or $\mathbb{C}$, and suppose that $g$ does not vanish identically. The following are equivalent: 
(1) The order of $g(a, y)$ (as a function of $y$ ) at $y=0$ equals $m$ for all fixed a sufficiently small.

(2) For some function $u$ analytic near the origin with $u(0,0) \neq 0$ we have $g(x, y)=$ $y^{m} u(x, y)$ for all $(x, y)$ sufficiently small.

(3) $g$ is order-invariant in the hyperplane $y=0$ near the origin and this order is equal to $m$.

Proof. First we show that (1) implies (2). Suppose that $g(\alpha, y)$ is of order $m$ at $y=0$ for $\alpha$ within box $B$ about the origin. Expand $g$ about the origin as the following iterated series:

$$
g(x, y)=g_{0}(x)+g_{1}(x) y+g_{2}(x) y^{2}+\cdots .
$$

Take $(a, 0) \in B$. By assumption $g_{i}(a)=0$ for all $i<m$ and $g_{m}(a) \neq 0$. Setting

$$
u(x, t)=g_{m}(x)+g_{m+1}(x) y+g_{m+2}(x) y^{2}+\cdots,
$$

we have $g(x, y)=y^{m} u(x, y)$, and $u(0,0) \neq 0$, as required.

Our proof above that (1) implies (2) could be easily adapted to show that (3) implies (2). That (2) implies (1) and (3) is straightforward.

Remark 4.5. One can adapt easily the above proof to show that the conditions (1)-(3) also are equivalent to

(4) $g$ is valuation-invariant in the hyperplane $y=0$ near the origin, and this valuation is equal to $(0, m)$ (where 0 denotes $n-1$ zeros).

We do not use this result in this paper.

\section{Proof of LAZARD'S MAIN Claim}

We shall need to sharpen slightly the definition of Lazard delineability given in Section 2. First recall that a $k$-dimensional analytic submanifold of $\mathbb{R}^{n-1}$ is a nonempty subset $S$ which looks locally like $\mathbb{R}^{k}$. That is, for each point $p$ of $S$, there is an analytic coordinate system $\left(\hat{x}_{1}, \ldots, \hat{x}_{n-1}\right)$ about $p$ such that near $p, S$ is the intersection of the $n-1-k$ hyperplanes $\hat{x}_{k+1}=0, \ldots, \hat{x}_{n-1}=0$ in the local coordinate system [31, 32]. With this notation, we say that $\left(\hat{x}_{1}, \ldots, \hat{x}_{k}\right)$ are local coordinates on $S$ near $p$. A function $\theta: S \rightarrow \mathbb{R}$ is said to be analytic if near every point $p$ of $S$, there are local coordinates on $S$ with respect to which $f$ is analytic (near the origin in $\mathbb{R}^{k}$ ). We say that an $n$-variate real polynomial $f$ is Lazard analytic delineable on a submanifold $S$ of $\mathbb{R}^{n-1}$ if conditions (1), (2) and (3) of Definition 2.10 are satisfied, where the continuous functions $\theta_{1}<\ldots<\theta_{k}$ from $S$ to $\mathbb{R}$ are moreover analytic. The major effort of this section is to prove the following result; the Lazard claim is then an easy consequence.

Theorem 5.1. Let $f\left(x, x_{n}\right) \in \mathbb{R}\left[x, x_{n}\right]$ have positive degree $d$ in $x_{n}$, where $x=\left(x_{1}, \ldots, x_{n-1}\right)$. Let $D(x), l(x)$ and $t(x)$ denote the discriminant, leading coefficient and trailing coefficient (that is, the coefficient independent of $x_{n}$ ) of $f$, respectively, and suppose that each of these polynomials is nonzero (as an element of $\mathbb{R}[x]$ ). Let $S$ be a connected analytic submanifold of $\mathbb{R}^{n-1}$ in which $D, l$ and $t$ are all valuation-invariant. Then $f$ is Lazard analytic delineable on $S$, hence $f$ is valuation-invariant in every Lazard section and sector over $S$. Moreover, the same conclusion holds for the polynomial $f^{*}\left(x, x_{n}\right)=x_{n} f\left(x, x_{n}\right)$. 
A special form of Lazard's main claim, in which $S$ is assumed to be a connected submanifold of $\mathbb{R}^{n-1}$, and each element of $A$, an irreducible basis, is concluded to be Lazard analytic delineable on $S$ etc., follows from the above theorem by Proposition 3.4. The details are provided in Subsection 5.3 below. This special form of Lazard's main claim is sufficient to validate Lazard's CAD method, as outlined in Section 2.

Our task now is to prove Theorem 5.1. To do this we first need to investigate transforming the valuation of $f$ at a point $p$ into the order of $f$ along a curve passing through $p$.

5.1. Transforming the valuation. For a pair of vectors $\mathbf{c}, \mathbf{v}$ we denote by $\langle\mathbf{c}, \mathbf{v}\rangle$ their scalar product $\langle\mathbf{c}, \mathbf{v}\rangle=\sum_{i} c_{i} v_{i}$. Let $V \subset \mathbb{N}^{n}$ be a non-empty family. We say that an $n$-tuple of positive integers $\mathbf{c}=\left(c_{1}, \ldots, c_{n}\right) \in\left(\mathbb{N}^{*}\right)^{n}$ is an evaluator for $V$ if for every $i=1, \ldots, n-1$ we have

$$
c_{i} \geq 1+\max _{\mathbf{v} \in V} \sum_{j>i} c_{j} v_{j} .
$$

The above inequality is intended to mean that the subset $\left\{\sum_{j>i} c_{j} v_{j} \mid \mathbf{v} \in V\right\}$ of $\mathbb{N}$ is finite and, if so, $c_{i}$ strictly exceeds the maximum element of this set. Such a c always exists for any given finite $V \subset \mathbb{N}^{n}$, but may or may not exist if $V$ is infinite. Indeed, if $V$ is finite, we may choose $c_{n}$ arbitrarily and then use (6) to define $c_{n-1}, c_{n-2}, \ldots$ recursively. This is enough for us because we shall typically need an evaluator for a set $V$ of valuations of some $n$-variate polynomial $g$. Then $\left\{v_{\alpha}(g) \mid \alpha \in K^{n}\right\}$ is finite, in fact every i-th component $\left(v_{\alpha}(g)\right)_{i}$ of $v_{\alpha}(g)$ is bounded by the maximal exponent of $x_{i}^{\beta_{i}}$ that appears in $g$.

Remark 5.2. If $\mathbf{c}=\left(c_{1}, \ldots, c_{n}\right)$ is an evaluator for $V$ then $\mathbf{c}^{\prime}=\left(c_{1}, \ldots c_{n-1}\right)$ is an evaluator for $V^{\prime}=\left\{\left(v_{1}, \ldots, v_{n-1}\right) \mid \exists_{v_{n}}\left(v_{1}, \ldots, v_{n}\right) \in V\right\}$.

Lemma 5.3. Let $V \subset \mathbb{N}^{n}$ be a non-empty family and let $\mathbf{c}=\left(c_{1}, \ldots, c_{n}\right) \in\left(\mathbb{N}^{*}\right)^{n}$ be an evaluator for $V$.

If $\mathbf{v} \in V, \mathbf{u} \in \mathbb{N}^{n}$, and $\mathbf{v}<_{\text {lex }} \mathbf{u}$ for the lexicographic order, then $\langle\mathbf{c}, \mathbf{v}\rangle\langle\langle\mathbf{c}, \mathbf{u}\rangle$. In particular, if $\mathbf{v}, \mathbf{u} \in V$ then $\langle\mathbf{c}, \mathbf{v}\rangle=\langle\mathbf{c}, \mathbf{u}\rangle$ if and only if $\mathbf{v}=\mathbf{u}$.

Proof. Suppose that $\mathbf{v}<_{l e x} \mathbf{u}$, that is there is $i$ such that $u_{k}=v_{k}$ for $k<i$ and $v_{i}<u_{i}$. Then

$$
\begin{aligned}
\langle\mathbf{c}, \mathbf{u}\rangle-\langle\mathbf{c}, \mathbf{v}\rangle & =c_{i}\left(u_{i}-v_{i}\right)+\sum_{j>i} c_{j}\left(u_{j}-v_{j}\right) \\
& \geq\left(u_{i}-v_{i}\right)+\left(c_{i}-1\right)\left(u_{i}-v_{i}-1\right)+\left[c_{i}-1-\sum_{j>i} c_{j} v_{j}\right]>0 .
\end{aligned}
$$

(The strict inequality uses the fact that the term in brackets is nonnegative since $\mathbf{c}$ is an evaluator for $V$ and $\mathbf{v} \in V$.)

The following result allows us to transform the Lazard valuation $v_{p}(f)$ into the order of $f$ at $y=0$ along a monomial curve of the form $K \ni y \rightarrow p+\left(y^{c_{1}}, \ldots, y^{c_{n}}\right)$.

Proposition 5.4. Let $f \in K\left[x_{1}, \ldots, x_{n}\right], f \neq 0, p \in K^{n}$. With $V \subset \mathbb{N}^{n}$ a non-empty family, suppose that $v_{p}(f) \in V$ and that $\mathbf{c}=\left(c_{1}, \ldots, c_{n}\right) \in\left(\mathbb{N}^{*}\right)^{n}$ is an evaluator for $V$. Then $f\left(p+\left(y^{c_{1}}, \ldots, y^{c_{n}}\right)\right)$ is not identically equal to zero and its order at $y=0$ equals $\left\langle\mathbf{c}, v_{p}(f)\right\rangle$. 
Proof. Write $f(x)=\sum_{\mathbf{v} \in \mathbb{N}^{n}} a_{\mathbf{v}}(x-p)^{\mathbf{v}}$ and denote $\Lambda=\left\{\mathbf{v} ; a_{\mathbf{v}} \neq 0\right\}$. Then

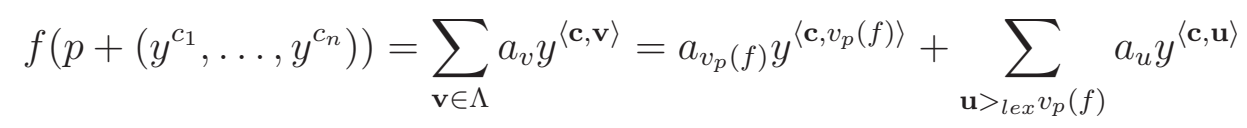

and the proposition follows from Lemma 5.3.

Proposition 5.5. Let $f \in K\left[x_{1}, \ldots, x_{n}\right], f \neq 0, \alpha \in K^{n-1}$. Let $\mathbf{v}=\left(v_{1}, \ldots, v_{n-1}\right)$ be the Lazard valuation of $f$ on $\alpha$. With $V^{\prime} \subset \mathbb{N}^{n-1}$ a non-empty family, suppose that $\mathbf{v} \in V^{\prime}$ and that $\mathbf{c}^{\prime}=\left(c_{1}, \ldots, c_{n-1}\right)$ is an evaluator for $V^{\prime}$. Then, the following formula holds:

$$
f\left(\alpha+\left(y^{c_{1}}, \ldots, y^{c_{n-1}}\right), x_{n}\right)=y^{\left\langle\mathbf{c}^{\prime}, \mathbf{v}\right\rangle}\left(f_{\alpha}^{\mathbf{v}}\left(x_{n}\right)+y R\left(y, x_{n}\right)\right),
$$

with $R \in K\left[y, x_{n}\right]$.

Proof. This follows from the definition of Lazard evaluation on $\alpha$.

We have also a parametrised version of the above result.

Proposition 5.6. Let $f \in K\left[x_{1}, \ldots, x_{n}\right], f \neq 0, S \subset K^{n-1}$. Let $\mathbf{v}=\left(v_{1}, \ldots, v_{n-1}\right)$ be the minimal value of Lazard valuation of $f$ on $\alpha \in S$. With $V^{\prime} \subset \mathbb{N}^{n-1}$ a non-empty family, suppose that $\mathbf{v} \in V^{\prime}$ and that $\mathbf{c}^{\prime}=\left(c_{1}, \ldots, c_{n-1}\right)$ is an evaluator for $V^{\prime}$. Then, the following formula holds for all $\alpha \in S$ :

$$
f\left(\alpha+\left(y^{c_{1}}, \ldots, y^{c_{n-1}}\right), x_{n}\right)=y^{\left\langle\mathbf{c}^{\prime}, \mathbf{v}\right\rangle}\left(f_{\alpha}^{\mathbf{v}}\left(x_{n}\right)+y R\left(\alpha, y, x_{n}\right)\right),
$$

with $f_{\alpha}^{\mathbf{v}}\left(x_{n}\right) \in K\left[\alpha, x_{n}\right], R \in K\left[\alpha, y, x_{n}\right]$.

Proof. This follows from the fact that the coefficient $f_{\alpha}^{u}$ in the expansion (1) is a polynomial in $\left(\alpha, x_{n}\right)$. (If $K$ is of characteristic zero then it is equal to $\frac{1}{u_{1} ! \cdots u_{n-1} !} \frac{\partial^{u_{1}+\cdots+u_{n-1}} f}{\partial x_{1}^{u_{1}} \cdots \partial x_{n-1}^{u_{n-1}}}$.) By assumption on $\mathbf{v}$, if $u<_{l e x} \mathbf{v}$ then $f_{\alpha}^{u}$ vanishes identically for $\alpha \in S$.

5.2. Proof of Theorem 5.1. The proof is based on the propositions stated in the previous subsection (especially Proposition [5.6) and the Puiseux with parameter theorem that we recalled in the previous section.

Recall that, in the statement of the theorem to be proved, $x$ denotes the $(n-1)$-tuple $\left(x_{1}, \ldots, x_{n-1}\right)$. Write

$$
f\left(x, x_{n}\right)=a_{d}(x) x_{n}^{d}+a_{d-1}(x) x_{n}^{d-1}+\cdots+a_{0}(x) .
$$

(Then $a_{d}(x)=l(x)$ and $a_{0}(x)=t(x)$.) We fix positive integers $c_{1}, \ldots, c_{n-1}$ that satisfy the following properties. Firstly, we want $\mathbf{c}=\left(c_{1}, \ldots, c_{n-1}\right)$ to be an evaluator for the set $V_{S}$ of Lazard valuations of $f$ on $p \in S$. Secondly we require that $\mathbf{c}$ should be an evaluator for $V_{g_{i}}=\left\{v_{p}\left(g_{i}\right): p \in S\right\}$, for $1 \leq i \leq 3$, where $g_{1}(x)=D(x), g_{2}(x)=a_{d}(x)$ and $g_{3}(x)=a_{0}(x)$. Since $V_{S}$ and the $V_{g_{i}}$ are finite such $c_{1}, \ldots, c_{n-1}$ exist. Later in the course of the proof we may multiply all $c_{i}$ by a positive integer $N$. Then clearly the vector $\left(N c_{1}, \ldots, N c_{n-1}\right)$ still is an evaluator for $V_{S}$ and the $V_{g_{i}}$. We shall use test monomial curves, as in Proposition 5.4, in the proof. Indeed we translate the assumed Lazard invariance of $D, a_{d}$, and $a_{0}$ for $p \in S$ near a fixed point $p_{0}$ of $S$ into the invariance of the order of these polynomials at $y=0$ along a suitable monomial curve parametrized by $y$ and passing through $p$, for $p \in S$ near $p_{0}$. This sets the stage for application of Lemma 4.4 followed by the Puiseux with parameter theorem. The details follow. 
By the assumptions of Theorem 5.1, $g_{i}$ is valuation-invariant in $S$, for $1 \leq i \leq 3$. Consider $\psi: S \times \mathbb{R} \rightarrow \mathbb{R}^{n-1}$ defined by

$$
\psi(p, y)=p+\left(y^{c_{1}}, \ldots, y^{c_{n-1}}\right)
$$

and $g_{i}(\psi(p, y)), 1 \leq i \leq 3$. By Proposition 5.4, for $i$ and $p$ fixed, $g_{i}(\psi(p, y))$, as a function of $y \in \mathbb{R}$, has order $k_{i}:=\left\langle\mathbf{c}, v_{p}\left(g_{i}\right)\right\rangle$ at $y=0$. Since $g_{i}$ is valuation-invariant in $S$, this order is independent of $p \in S$. Therefore, by Lemma 4.4, applied with respect to suitable local coordinates on $S \times \mathbb{R}$ near $\left(p_{0}, 0\right), g_{i}(\psi(p, y))$ as an analytic function is divisible by $y^{k_{i}}$ with the quotient analytic and non-vanishing in a neighbourhood $U_{S} \times U^{\prime}$ of $\left(p_{0}, 0\right)$ in $S \times \mathbb{R}$. Let

$$
f_{\psi}(p, y, z)=f(\psi(p, y), z)=a_{d}(\psi(p, y)) z^{d}+a_{d-1}(\psi(p, y)) z^{d-1}+\cdots+a_{0}(\psi(p, y)) .
$$

Since $a_{d}(\psi(p, y))$ is not vanishing identically, the degree in $z$ of $f_{\psi}$ equals $d$ and the discriminant $D_{\psi}(p, y)$ of $f_{\psi}$ equals $D(\psi(p, y))$. Therefore we may apply to $f_{\psi}$, localised at $\left(p_{0}, 0\right) \in S \times \mathbb{R}$, and after complexification, the Puiseux with parameter theorem in the form given by Corollary 4.2. Then we use the conclusion of Corollary 4.2 to show the Lazard delineability of $f$ over a neighbourhood of $p_{0}$ in $S$. Now we present in detail this argument.

Where $k$ denotes the dimension of $S$, we choose local coordinates $(\hat{x}, y)=\left(\hat{x}_{1}, \ldots, \hat{x}_{k}, y\right)$ on $S \times \mathbb{R}$ near $\left(p_{0}, 0\right)$. We denote by $\hat{f}_{\psi}(\hat{x}, y, z)$ the polynomial $f_{\psi}$ expressed in these coordinates, and by $\hat{a}_{i}$ and $\hat{D}_{\psi}$ its $i$ th coefficient and discriminant, respectively. Then the $\hat{a}_{i}$ are analytic in a neighbourhood of the origin in $\mathbb{R}^{k+1}$. We denote by $\hat{a}_{i}$ also the complexification of $\hat{a}_{i}$, that is, the unique complex analytic extension of $\hat{a}_{i}$ to a neighbourhood of the origin in $\mathbb{C}^{k+1}$.

Hence, in a suitable polydisk $U_{\varepsilon, r}$ in $\mathbb{C}^{k+1}, \hat{f}_{\psi}\left(\right.$ and $\left.\hat{a}_{d}, \hat{a}_{0}, \hat{D}_{\psi}\right)$ satisfy the hypotheses of Corollary 4.2 (as was observed above). Therefore, by this Corollary, we have

$$
t^{d m_{0}} \hat{f}_{\psi}\left(\hat{x}, t^{N}, z\right)=\hat{a}_{d}\left(\hat{x}, t^{N}\right) \prod_{i}\left(t^{m_{0}} z-t^{m_{i}} \hat{u}_{i}(\hat{x}, t)\right)
$$

in $U_{\varepsilon, r^{1 / N}}$, for suitable integers $N>0$ and $m_{i} \geq 0$, and suitable functions $\hat{u}_{i}(\hat{x}, t)$ analytic and nonvanishing in the specified polydisk.

Now we may write $\hat{a}_{d}\left(\hat{x}, t^{N}\right)=t^{m_{0}} \hat{\tilde{a}}_{d}\left(\hat{x}, t^{N}\right)$, with $\hat{\tilde{a}}_{d}\left(\hat{x}, t^{N}\right)$ nonvanishing in the specified polydisk, as in the proof of the Corollary just cited. Hence, in our original coordinates, we have

$$
t^{(d-1) m_{0}} f_{\psi}\left(p, t^{N}, z\right)=\tilde{a}_{d}\left(p, t^{N}\right) \prod_{i}\left(t^{m_{0}} z-t^{m_{i}} u_{i}(p, t)\right)
$$

in $U_{S} \times U^{\prime}$, after suitable refinement of this neighbourhood if necessary, where the functions $\tilde{a}_{d}, u_{i}$ are analytic and nonvanishing in $U_{S} \times U^{\prime}$. Moreover, again by the conclusion of Corollary 4.2, each difference $t^{m_{i}} u_{i}(p, t)-t^{m_{j}} u_{j}(p, t)$ for $i \neq j$ equals a power of $t$ times a nowhere vanishing function. Let $\mathbf{v}$ be the minimum of valuations of $f$ on $p$ for $p \in U_{S}$. By (8)

$$
f_{\psi}\left(p, t^{N}, z\right)=t^{N\langle\mathbf{c}, \mathbf{v}\rangle}\left(f_{\alpha}^{\mathbf{v}}(z)+t^{N} R\left(p, t^{N}, z\right)\right) .
$$

By comparing (13) and (12) we see that $N\langle\mathbf{c}, \mathbf{v}\rangle=m_{0}(1-d)+\sum_{i} \max \left\{m_{0}, m_{i}\right\}$. (The nonvanishing of the $u_{i}$ is used here.) Therefore the Lazard valuation of $f$ on $p$ is independent 
of $p \in U_{S}$, and the Lazard evaluation of $f$ at $p$ equals

$$
f_{p}^{\mathbf{v}}(z)=\tilde{a}_{d}(p, 0) z^{\nu} \prod_{m_{i}=m_{0}}\left(z-u_{i}(p, 0)\right) \prod_{m_{i}<m_{0}} u_{i}(p, 0),
$$

where $\nu$ equals the number of $i$ with $m_{i}>m_{0}$. By Corollary 4.2, each difference $u_{i}(p, 0)-$ $u_{j}(p, 0)$, for $i, j$ such that $m_{i}=m_{j}=m_{0}$, is either non-vanishing for all $p \in U_{S}$ or identically equal to zero in $U_{S}$. Hence, by the last part of Corollary 4.2 (concerning the complex conjugation invariance of the $\left.u_{i}\right)$, each $u_{i}(p, 0)$ is either real for all $p$, if $u_{i}(p, 0) \equiv \bar{u}_{i}(p, 0)$, or nonreal for all $p$, otherwise. Thus we may take as the $\theta_{j}(p)$ of $(2)$ of Definition 2.10 those $u_{i}(p, 0)$ that are real, and complete them by $\theta(p) \equiv 0$ if $\nu$ of the equation above is strictly positive. This shows that $f$ is Lazard analytic delineable on $U_{S}$. The Lazard analytic delineability of $f$ on the whole of $S$ then follows from the connectedness of $S$.

It remains to address the proof of the second conclusion of Theorem 5.1, which asserts the Lazard analytic delineability of $f^{*}=x_{n} f$ on $S$. Recall that the functions $u_{i}(p, 0)$ which determine part of the variety of $f_{p}^{\mathbf{v}}(z)$ for $p$ in $S$ near $p_{0}$ vanish nowhere near $p_{0}$. If $\nu>0$ the remaining part of the variety of $f_{p}^{\mathbf{v}}(z)$ is given by the function $z \equiv 0$. Therefore each real root function $\theta$ of $f$ on $S$ satisfies the following: either $\theta(p)<0$ for all $p$, or $\theta(p)=0$ for all $p$, or $\theta(p)>0$ for all $p$. Therefore $f^{*}=x_{n} f$ is Lazard analytic delineable on $S$.

An analogue of our main theorem, with slightly different hypotheses but the same conclusion, is true. The hypotheses of this analogue are that the discriminant $D(x)$ is valuation invariant in $S$ and the leading coefficient $l(x)$ is nonvanishing in $S$. No assumption about the trailing coefficient $t(x)$ is needed. The proof of this analogue is similar to (and simpler than) the proof of our main theorem presented above, except that Theorem 4.1 is used instead of its corollary, after dividing through by the leading coefficient of $f_{\psi}$. This analogue could be used to enhance the practical efficiency of Algorithm 1 in cases where some polynomial elements of the input set $A$ have leading coefficients which vanish nowhere in $\mathbb{R}^{n-1}$. As the following examples show we cannot drop the assumption about $t(x)$ in general.

Example 5.7. With $\left(x_{1}, x_{2}, x_{3}\right)=(x, y, z)$, consider $f(x, y, z)=y^{2} z^{2}-y(2 x+y) z+x(x+y)$ and let $S$ be the $x$-axis. Then $D(x, y)=y^{4}, a_{2}(x, y)=y^{2}$ and hence both $D$ and $a_{2}$ are valuation invariant on $S$. Nevertheless $f$ is not Lazard delineable on $S$. Indeed, $f$ vanishes identically over $x=y=0$ but not over the generic point of $S$. An even simpler example is furnished by $f(x, y, z)=y z-x$, with the same $S$.

We illustrate the construction in the proof using two examples. For both examples we take $n=4$ and $\left(x_{1}, x_{2}, x_{3}, x_{4}\right)=(x, y, z, w)$.

Example 5.8. Let $f(x, y, z, w)=y w^{2}+x w-y z^{2}$ and let $S \subset \mathbb{R}^{3}$ be the positive direction of the $z$-axis (not including the origin). The discriminant $D(x, y, z)=x^{2}+4 y^{2} z^{2}$ vanishes identically on $S$. The valuations of $D$, the leading coefficient $a_{2}=y$, and the trailing coefficient $a_{0}=-y z^{2}$, at $(0,0, z), z>0$, are equal to $(0,2,0),(0,1,0)$, and $(0,1,0)$ respectively. (At the origin they are $(0,2,2),(0,1,0)$, and $(0,1,2)$, so we do not include the origin in $S$.)

In order to detect the decomposition of $S \times \mathbb{R}$ into the valuation (of $f$ ) invariant subsets we may take $c_{1}=3, c_{2}=c_{3}=1$, so that the condition (6) is satisfied for the sets $V_{g}=$ $\left\{v_{p}(g): p \in S\right\}$, where $g$ equals $D, a_{2}$, and $a_{0}$, and for the set $V_{S}$ of Lazard valuations of $f$ 
on $p \in S$. In this case it means that (6) is satisfied for $(0,1,0)$ and $(0,2,0)$. The function $f_{\psi}$ is then given by

$$
f_{\psi}(p, s, w)=s w^{2}+s^{3} w-s(z+s)^{2}=s\left(w^{2}+s^{2} w-(z+s)^{2}\right)
$$

where $p=(0,0, z)$. (Note that we use the symbol $s$ to denote the second argument of $f_{\psi}$, since $y$ already denotes $x_{2}$.) This case is particularly simple since by dividing by $s$ and then setting $s=0$ we obtain a polynomial of the same degree as $f$. This polynomial $w^{2}-z^{2}$ equals the Lazard evaluation polynomial $f_{p}(w), p=(0,0, z)$, and its zeros give the decomposition of $S \times \mathbb{R}$ into the valuation invariant subsets: $v_{(p, w)}(f)=(0,1,0,1)$ if $w^{2}=z^{2}>0$ and $v_{(p, w)}(f)=(0,1,0,0)$ if $w^{2} \neq z^{2}, z>0$. (If $w=z=0$ then $v_{(p, w)}(f)=(0,1,0,2)$.)

A similar but more complicated example is $f(x, y, z, w)=x w^{2}+y z w-x$ with the same $S$ and similar discriminant $D(x, y, z)=y^{2} z^{2}+4 x^{2}$. Then the Lazard evaluation polynomial $f_{p}(w)=z w$ for $p=(0,0, z), z>0$, (here $z$ is treated as a constant), is of degree strictly smaller than the degree of $f$. We may take again $c_{1}=3, c_{2}=c_{3}=1$. The function $f_{\psi}$ (again with $s$ denoting the second argument) is given by

$$
f_{\psi}(p, s, w)=s^{3} w^{2}+s w(z+s)-s^{3}=s\left(s^{2} w^{2}+w(z+s)-s^{2}\right) .
$$

The polynomial in parentheses has two roots for $s \neq 0$. One of them tends to 0 as $s$ tends to 0 and the other one tends to infinity. In the formula (12), after dividing through by $t^{(d-1) m_{0}}$, this latter root of $f_{\psi}(p, t, w)$ has a strictly negative exponent $m_{i}-m_{0}$. The exponent associated to the first root is strictly positive.

5.3. Derivation of Lazard's main claim from Theorem 5.1. We show how the special form of Lazard's main claim described just after the statement of Theorem 5.1 is deduced from Theorem 5.1. We essentially adapt the proof of Theorem 3.1 of 31 in the following way. Let $x$ denote $\left(x_{1}, \ldots, x_{n-1}\right)$. Let $A=\left\{f_{1}, f_{2}, \ldots, f_{m}\right\}$ be a finite irreducible basis in $R_{n}$, where $n \geq 2$, and let $S$ be a connected analytic submanifold of $\mathbb{R}^{n-1}$. Suppose that each element of $P_{L}(A)$ is valuation-invariant in $S$. If $\pm x_{n} \notin A$, put $f=f_{1} f_{2} \cdots f_{m}$. Otherwise put $f=f_{1} f_{2} \cdots f_{m} / x_{n}$. Then $f$ is (up to sign) the product of those elements of $A$ whose trailing coefficients are nonzero. Hence the trailing coefficient $t(x)$ of $f$ is nonzero, and is valuation-invariant in $S$, by Proposition [3.4. Similarly, the leading coefficient $l(x)$ of $f$ is valuation-invariant in $S$. Using the well-known expression for the discriminant of a polynomial product and Proposition 3.4, we see also that the discriminant $D(x)$ of $f$ is nonzero and valuation-invariant in $S$. Hence, by the first conclusion of Theorem 5.1, $f$ is Lazard analytic delineable on $S$. If $\pm x_{n} \notin A$, we may use this property to deduce the desired conclusions of Lazard's main claim. If $\pm x_{n} \in A$, then by the second conclusion of Theorem $5.1, f^{*}=x_{n} f=f_{1} f_{2} \cdots f_{m}$ is Lazard analytic delineable on $S$, and the desired conclusions of Lazard's main claim follow from this property of $f^{*}$.

5.4. Proof of Lazard's original claim. In this subsection we provide a proof of the original claim of Lazard, as stated in Section 2. We first state a corollary of Theorem 5.1:

Corollary 5.9. Suppose $f\left(x, x_{n}\right) \in \mathbb{R}\left[x, x_{n}\right]$ satisfies the assumption of Theorem [5.1. Let $S$ be a connected subset of $\mathbb{R}^{n-1}$ in which $D, l$ and $t$ are all valuation-invariant. Then $f$ is Lazard delineable on $S$ and is valuation invariant in every section and sector of $f$ over $S$.

Proof. This corollary follows from Theorem 5.1 by standard arguments of semialgebraic geometry: stratifications and the curve selection lemma. 
First we note that there is a finite semialgebraic stratification $\sqcup_{i} T_{i}=\mathbb{R}^{n-1}$ such that $D, l$ and $t$ are all valuation-invariant on each stratum. Here, by definition of stratification, each $T_{i}$ is a connected locally closed semialgebraic subset and an analytic submanifold of $\mathbb{R}^{n-1}$ and any two strata satisfy the frontier condition: if $T_{i} \cap \bar{T}_{j} \neq \emptyset$ then $T_{i} \subset \bar{T}_{j}$. The existence of such a stratification follows from general theory of semialgebraic sets, see e.g. Proposition 9.1.8 of [6] or Proposition 2.5.1 of [4].

Now for $S \subset \mathbb{R}^{n-1}$ as in the assumption of corollary we consider $S_{1}=\sqcup_{i \in \Lambda} T_{i}$, the union of all strata intersecting $S$. Clearly $S_{1}$ is connected, $D, l$ and $t$ are all valuation-invariant in $S_{1}$, and Lazard delineability on $S_{1}$ implies Lazard delineability on $S$. Thus we may replace $S$ by $S_{1}$.

In order to show Lazard delineability on a connected union of strata it suffices to show it on $S$ of the form $S=T_{i} \cup T_{j}$ with $T_{i} \subset \bar{T}_{j}$. Thus suppose that $S=T_{i} \cup T_{j}$ and that $D, l$ and $t$ are valuation-invariant on $S$. We show that $f$ is delineable on $S$. For this it is enough to show that the Lazard valuation on $\alpha$ is independent of $\alpha \in S$, that the number of sections of $f_{\alpha}$ over $T_{i}$ and $T_{j}$ coincide, that these sections are given by functions continuous on whole $S$, and finally that their multiplicities as roots of $f_{\alpha}$ are the same on $T_{i}$ and $T_{j}$. By standard arguments based on the curve selection lemma, see e.g. Proposition 8.1.13 of [6], it suffices to show all these claims over $p([0, \varepsilon))$, where $p(\tau):(-\varepsilon, \varepsilon) \rightarrow S$ is an arbitrary real analytic curve such that $p(0) \in T_{i}$ and $p(\tau) \in T_{j}$ for $\tau>0$. By replacing $\tau$ by $\tau^{2}$ we may assume that $p(\tau) \in T_{j}$ for $\tau \neq 0$. Then we follow the main steps of the proof of Theorem 5.1. Let $\psi:(-\varepsilon, \varepsilon) \times \mathbb{R} \rightarrow \mathbb{R}^{n-1}$ be defined by

$$
\psi(\tau, y)=p(\tau)+\left(y^{c_{1}}, \ldots, y^{c_{n-1}}\right),
$$

where $\left(c_{1}, \ldots, c_{n-1}\right)$ is an evaluator for the sets $V_{g}=\left\{v_{p}(g): p \in S\right\}$, with $g(x)=D(x), l(x)$ and $t(x)$, and an evaluator for the set $V_{S}$ of Lazard valuations of $f$ on $p \in S$. Since $D, l$ and $t$ are valuation-invariant on the image of $p(\tau), f_{\psi}(\tau, y, z)=f(\psi(\tau, y), z)$ satisfies the assumptions of Puiseux with parameter theorem, Corollary 4.2, Then the proof of Theorem 5.1 shows that $f$ is Lazard delineable on the image of $p(\tau)$, and hence, by the curve selection lemma, on $S$.

Lazard's original claim follows from the above corollary by analogy with the argument presented in Subsection 5.3 above.

\section{Conclusion}

We first summarise the work reported herein. We presented the results of our investigation of Lazard's proposed CAD method, including both his proposed projection and valuation. In [36] we already found that Lazard's projection is valid for CAD construction for welloriented polynomial sets. In the present paper (Section 5) Lazard's main claim is proved using his valuation. A consequence of this result is that Lazard's CAD method is valid, with no well-orientedness restriction.

There are immediate consequences of our main result for certain related works on projection in CAD. For example, as similarly mentioned in [36] (Section 4), our main result could be readily adapted to obtain an analogue of the theorem of [33] concerning the reduction of projection sets in CAD-based quantifier elimination in the presence of equational constraints. A further significant potential benefit of Lazard's method is that it may permit greater simplifications and improvements to projection for such problems. Indeed, the newly validated 
approach which underpins improved projection using valuation invariance may yield better results concerning the use of so-called propagated constraints for such problems [34].

It is natural to ask how Lazard's CAD method compares with other reduced projection CAD algorithms with respect to efficiency and other criteria. As mentioned, Lazard's method is more general than those of McCallum [30, 31, 32] and Brown [8] in the sense that the latter algorithms fail for non-well-oriented input sets $A$. The algorithm of [30] was subjected to a theoretical computing time analysis which was broadly based upon that provided by Collins [15] for his original CAD algorithm. The overall conclusion was that the method of [30] remains of doubly exponential worst case time complexity in the number of variables $n$, as is the case for Collins' original CAD, though the double exponent in the computing time bound is reduced. Still, for every fixed $n$, both algorithms have a polynomial worst case computing time bound. (Interested readers are referred to the very recent paper [7] which contains an improved exposition of the complexity analysis of [30].) To our knowledge, no computing time analysis of Brown's method, nor that of Lazard, has yet been published. Nonetheless, given that both sets $P_{B M}(B)$ and $P_{L}(B)$ contain the discriminants, and resultants of pairs of distinct elements, of an irreducible basis $B$, it is likely that the computing times of the methods of both Brown and Lazard remain doubly exponential in $n$, perhaps with slight improvements to the double exponent, relative to McCallum's method.

Further work could usefully be done in a number of directions. As mentioned and elaborated in [36], it will be interesting to compare experimentally the Brown-McCallum projection [8] with the Lazard projection. As mentioned above, it would be worthwhile to try to extend the theory of equational constraints, especially the use of propagated constraints, with the Lazard projection. Similarly, re-examination of the theory of bi-equational constraints [9, 10] in the context of Lazard's projection and valuation may be fruitful. Re-visiting ideas for practical improvements suggested in [26] could be beneficial.

Another inportant direction will be to understand the topological and geometric structure of the output of the CAD algorithm. This concerns all the methods, not only the Lazard one presented in this paper, see [28]. To study it will be interesting to use the new ideas proposed in a recent paper on Zariski equisingularity and stratifications [37.

\section{Appendix. Proof of Puiseux with parameter theorem}

In this section, for the reader's convenience, we present a concise proof of Theorem 4.1. This proof is based on the classical theory of complex analytic functions and uses a parametrized version of the Riemann removable singularity theorem. The proof we present below is due to Łojasiewicz and Pawłucki, see [39]. For a similar approach, with slightly different details, see 38] Proposition 2.1.

Let us first recall the basic notation: $U_{\varepsilon, r}=U_{\varepsilon} \times U_{r}$, where $U_{\varepsilon}=\left\{x=\left(x_{1}, \ldots, x_{k}\right) \in \mathbb{C}^{k}\right.$ : $\left.\left|x_{i}\right|<\varepsilon_{i}, \forall i\right\}, U_{r}=\{y \in \mathbb{C}:|y|<r\}$. We also denote the punctured disc $U_{r} \backslash\{0\}$ by $U_{r}^{*}$.

Proof of Theorem 4.1. Consider the polynomial in $z$,

$$
P(x, w, z):=f\left(x, e^{2 \pi i w}, z\right),
$$

whose coefficients $a_{i}\left(x, e^{2 \pi i w}\right)$ are analytic on $U_{\varepsilon} \times H$, where $H=\{w: 2 \pi \operatorname{Im}(w)>-\ln r\}$. By assumption the discriminant $D_{P}(x, w)=D_{f}\left(x, e^{2 \pi i w}\right)$ does not vanish on $U_{\varepsilon} \times H$ and 
hence $P$ admits global complex analytic roots

$$
\tilde{\xi}_{1}(x, w), \cdots, \tilde{\xi}_{d}(x, w) .
$$

(If $D_{P}(x, w)$ is nonzero then the equation $P=\partial P / \partial z=0$ has no solution. Therefore the local solutions $\tilde{\xi}(x, w)$ of $P=0$ are analytic by the implicit function theorem. They are well-defined global analytic functions because $U_{\varepsilon} \times H$ is contractible.)

The coefficients of $P$ are periodic: $P(x, w+1, z)=P(x, w, z)$. Hence for each root $\tilde{\xi}_{i}(x, w)$ there is another root $\tilde{\xi}_{\varphi(i)}(x, w)$ such that $\tilde{\xi}_{i}(x, w+1)=\tilde{\xi}_{\varphi(i)}(x, w)$. The map $\varphi:\{1, \ldots, d\} \rightarrow\{1, \ldots, d\}$ is a permutation and hence $\varphi^{d !}=i d$. Therefore, for $N=d$,

$$
\tilde{\xi}_{i}(x, w+N)=\tilde{\xi}_{i}(x, w) .
$$

Thus there are analytic functions $\xi_{i}(x, t): U_{\varepsilon} \times U_{r^{1 / N}}^{*} \rightarrow \mathbb{C}$ such that $\tilde{\xi}_{i}(x, w)=\xi_{i}\left(x, e^{2 \pi i w / N}\right)$. Since $\xi_{i}(x, t)$ are roots of $f\left(x, t^{N}, z\right)$ they are bounded on $U_{\varepsilon_{0}} \times U_{r_{0}^{1 / N}}^{*}$, for every $\varepsilon_{0}<\varepsilon, r_{0}<r$. Hence they extend to functions analytic on $U_{\varepsilon} \times U_{r^{1 / N}}$ by Riemann's theorem on removable singularities, cf. [20] Theorem 3, p. 19.

\section{ACKNowledgements}

We acknowledge grants which supported visits to the University of Sydney by the second named author in 2015 and 2016 : Sydney University BSG, IRMA Project ID: 176623 and ANR project STAAVF (ANR-2011 BS01 009). We are grateful to Hoon Hong for kindly granting us permission to include some basic material from the technical report [35] in Section 3.

\section{REFERENCES}

[1] Abhyankar, S. S. On the ramification of algebraic functions. Amer. J. Math. 77, (1955), 575-592.

[2] Arnon, D. S., Collins, G. E., And McCallum, S. Cylindrical algebraic decomposition I: The basic algorithm. SIAM Journal on Computing 13, 4 (1984), 865-877.

[3] Atiyah, M. F., MacDonald, I. G. Introduction to Commutative Algebra. Addison-Wesley, Reading, 1969.

[4] Benedetti, R., Risler, J.-J. Algebraic and Semi-Algebraic Sets. Hermann, Paris, 1990.

[5] Becker, T., Weispfenning, V., And Kredel, H. Groebner Bases: A Computational Approach to Commutative Algebra. Corrected Second Printing. Springer, New York, 1998.

[6] Bochnak, J., Coste, M., Roy, M.-F., Real Algebraic Geometry. Springer-Verlag, Berlin Heidelberg, 1998.

[7] Bradford, R., Davenport, J., England, M., McCallum, S., Wilson, D. Truth table invariant cylindrical algebraic decomposition. Journal of Symbolic Computation 76, (2016), 1-35.

[8] Brown, C. W. Improved projection for cylindrical algebraic decomposition. Journal of Symbolic Computation 32, (2001), 447-465.

[9] Brown, C. W., And McCallum, S. On using bi-equational constraints in CAD construction. In Proceedings of ISSAC'05, Kauers, M. (Ed.), (2005), ACM Press, New York, pp. 76-83.

[10] Brown, C. W., And MCCAllum, S. On delineability of varieties in CAD-based quantifier elimination with two equational constraints. In Proceedings of ISSAC'09, May, J. (Ed.), (2009), ACM Press, New York, pp. 71-78.

[11] Brown, C., Kahoui, M., Novotni, D., Weber, A. Algorithmic methods for investigating equilibria in epidemic modelling. J. Symbolic Computation 41, (2006), 1157-1173.

[12] Busé, L., Mourrain, B. Explicit factors of some iterated resultants and discriminants. Math. Comp. 78, (2009), 345-386. 
[13] Caviness, B., And Johnson, J. R., Eds. Quantifier Elimination and Cylindrical Algebraic Decomposition. Texts and Monographs in Symbolic Computation. Springer-Verlag, 1998.

[14] Collins, G. E. Computer algebra of polynomials and rational functions. Amer. Math. Monthly 80, (1973), 725-755.

[15] Collins, G. E. Quantifier elimination for the elementary theory of real closed fields by cylindrical algebraic decomposition. In: Lecture Notes In Computer Science, Vol. 33, (1975), Springer-Verlag, Berlin, pp. 134-183. Reprinted in [13.

[16] Collins, G. E. Quantifier elimination by cylindrical algebraic decomposition - twenty years of progress. In: [13.

[17] Collins, G. E., And Hong, H. Partial cylindrical algebraic decomposition for quantifier elimination. Journal of Symbolic Computation 12, (1991), 299-328.

[18] Danilov, V. I. Valuation. Springer-Verlag Online Encyclopaedia of Math., 2010.

[19] Davenport, J.H., Bradford, R., England, M., Wilson, D. Program verification in the presence of complex numbers etc. In: SYNASC'12 (2012), IEEE, pp. 83-88.

[20] Gunning, H.C., Rossi, C. Analytic Functions of Several Complex Variables. AMS Chelsea Publishing, Providence, Rhode Island, 2009.

[21] Hensel, K. Ueber eine neue Theorie der algebraischen Funktionen zweier Variablen. Acta Math. 23, (1900), 339-416.

[22] Hong, H. An improvement of the projection operator in cylindrical algebraic decomposition. In: Proceedings of ISSAC'90, Watanabe, S. and Nagata, M. (Eds.), (1990), ACM Press, New York, pp. 261-264. Reprinted in [13].

[23] Hong, H., Liska, R., Steinberg, S. Testing stability by quantifier elimination. J. Symbolic Computation 24, (1997), 161-187.

[24] Jung, H. W. E. Darstellung der Funktionen eines algebraischen Koerpers zweier unabhaengigen Veraenderlichen $\mathrm{x}, \mathrm{y}$ in der Umgebung einer Stelle $\mathrm{x}=\mathrm{a}, \mathrm{y}=\mathrm{b}$. J. reine u. angewadte Math. 133, (1908), 289-314.

[25] Kaltofen, E. Factorization of polynomials. In: Computing, Supplementum 4: Computer Algebra Symbolic and Algebraic Computation, (1982), Springer Verlag, Vienna, pp. 95-113.

[26] LazARD, D. An improved projection for cylindrical algebraic decomposition. In Algebraic Geometry and its Applications, Bajaj, C.L. (ed.), Springer, New York, 1994.

[27] Lazard, D., McCallum, S. Iterated discriminants. Journal of Symbolic Computation 44, (2009), $1176-1193$.

[28] Lazard, D. CAD and Topology of Semi-Algebraic Sets Math.Comput.Sci., (2010) 4:93-112.

[29] Lipman, J., Teissier, B., Eds. Oscar Zariski: Collected Papers, Volume 4: Equisingularity on Algebraic Varieties. MIT Press, Cambridge, 1979.

[30] McCallum, S. An improved projection operation for cylindrical algebraic decomposition. PhD thesis, University of Wisconsin-Madison, 1984.

[31] McCallum, S. An improved projection operation for cylindrical algebraic decomposition of threedimensional space. Journal of Symbolic Computation 5, (1988), 141-161.

[32] McCallum, S. An improved projection operation for cylindrical algebraic decomposition. In [13].

[33] McCallum, S. On projection in CAD-based quantifier elimination with equational constraint. In: Proceedings of ISSAC'99, Dooley, S. (Ed.), (1999), ACM Press, New York, pp. 145-149.

[34] McCallum, S. On propagation of equational constraints in CAD-based quantifier elimination. In: Proceedings of ISSAC'01, Mourrain, B. (Ed.), (2001), ACM Press, New York, pp. 223-230.

[35] McCallum, S., And Hong, H. On Lazard's valuation and CAD construction. arXiv:1501.06563 [math.AG].

[36] McCallum, S., And Hong, H. On using Lazard's projection in CAD construction. Journal of Symbolic Computation 72, (2016), 65-81.

[37] Parusiński, A., And Paunescu, L. Arcwise Analytic Stratification, Whitney Fibering Conjecture and Zariski Equisingularity. Advances in Mathematics 309, (2017), 254-305.

[38] Parusiński, A., And Rond, G. The Abhyankar-Jung theorem. J. Algebra 365, (2012), 29-41. 
[39] Pawłucki, W. Le théorème de Puiseux pour une application sous-analytique. Bull. Polish Acad. Sci. Matha 32., (1984), 555-560.

[40] Schwartz, J., Sharir, M. On the "piano-movers" problem II: General techniques for computing topological properties of real algebraic manifolds. Adv. Appl. Math. 4, (1983), 298-351.

[41] Walker, R. J. Algebraic Curves. Springer-Verlag, New York, 1978.

[42] Weispfenning, V. Simulation and optimization by quantifier eleimination. J. Symbolic Computation 24, (1997), 189-208.

[43] Whitney, H. Complex Analytic Varieties. Addison-Wesley, Menlo Park, 1972.

[44] Zariski, O. Algebraic Surfaces. Springer-Verlag, New York-Heidelberg-Berlin, 1935.

[45] Zariski, O. Studies in equisingularity I. Equivalent singularities of plane algebroid curves. Amer. J. Math. 87, (1965), 507-536. Reprinted in [29].

[46] Zariski, O. Studies in equisingularity II. Equisingularity in codimension 1 (and characteristic zero). Amer. J. Math. 87, (1965), 972-1006. Reprinted in 29.

[47] Zariski, O. On equimultiple subvarieties of algebroid hypersurfaces. Proc. Nat. Acad. Sci., USA 72, 4 (1975), 1425-1426.

[48] Zariski, O., Samuel, P. Commutative Algebra Volume II. Springer-Verlag, New York, 1960.

Department of Computing, Macquarie University, NSW 2109, Australia

Univ. Nice Sophia Antipolis, CNRS, LJAD, UMR 7351, 06108 Nice, France

School of Mathematics and Statistics, University of Sydney, NSW 2006, Australia 